\title{
LETRAMENTO DIGITAL NO ENSINO DE INFORMÁTICA PARA IDOSOS
}

Literacy in the digital computer education for elderly

\author{
http://dx.doi.org/10.21116/2016.6
}

\section{TARALLO, Roberta dos Santos}

Faculdade de Jaguariúna e Faculdade Max Planck

SÉ, Elisandra Villela Gasparetto

Faculdade de Jaguariúna e Faculdade Max Planck

RESUMO: A presente pesquisa teve por objetivo identificar as motivações dos idosos para aprender informática e analisar as estratégias e práticas de uso do computador em sala de aula. Como método de estudo, os participantes voluntários, idosos regularmente matriculados no curso de informática, responderam ao questionário contendo questões objetivas. Os idosos avaliaram o curso de informática como ótimo e bom, expressando total satisfação. As motivações, as expectativas e os objetivos estão voltados para a atualização tecnológica e aquisição de conhecimento nesta área. As dificuldades assinaladas ainda são pontuais e correlacionadas ao manuseio do computador, ao entendimento e segurança sobre a tarefa e à necessidade de repetição e prática. Evidenciou-se que poucos se dedicam diariamente à atividade de informática fora da sala de aula. $\mathrm{O}$ modo de ensino-aprendizagem de informática para idosos é heterogêneo, observado na facilidade de alguns e na dificuldade de outros. As estratégias e práticas de uso do computador nas aulas de informática na Faculdade Aberta à Terceira Idade da Faculdade de Jaguariúna proporcionaram aos idosos oportunidades de participação em práticas sócio-comunicativas e de inclusão digital social.

Palavras-chaves: Educação, Informática, Idosos.

ABSTRACT: This research aimed to identify the motivations of elders to learn computer and analyze the strategies and computer use practices in the classroom. As a study method, volunteers participating elderly regularly enrolled in computer course, the questionnaire containing objective questions. The elderly evaluated the computer course as great and good, expressing total satisfaction. The motivations, expectations and objectives are focused on technological upgrading and acquisition of knowledge in this area. Difficulties still marked are specific and related to computer handling, the understanding and certainty of the task and the need for repetition and practice. It was evidenced that few elderly are dedicated daily at computer activity outside the classroom. The computer teaching-learning mode for the elderly is heterogeneous, noted the facility of some and the difficulty of others. Strategies and computer use practices in computer classes at Faculty for Third Age (Faculdade Aberta à Terceira Idade)" of the Faculty of Jaguariuna provided to older people opportunities to participate in social and communicative practices and social digital inclusion.

Keywords: Education, Computing, Elderly. 


\section{Introdução}

A tecnologia transforma as formas de comunicação e de interação, bem como o cotidiano das pessoas. Em sociedades que empregam a informatização, utilizam-se recursos eletrônicos em diversos contextos: domésticos, institucionais, empresariais, urbanos, rurais, midiáticos, culturais, dentre outros. O ser humano vive rodeado de tecnologias como a televisão, o rádio, o telefone fixo ou móvel, os caixas eletrônicos, as câmeras de monitoramento, os computadores em geral. Tais dispositivos são disseminadores de padrões e de valores sociais e culturais (FARAH et al., 2009; MÜLLER, 2012; ZENI et al., 2013; LIMA; ALMEIDA, 2015).

Quando se conecta ao mundo tecnológico e virtual, pode-se vivenciar um processo de construção e desconstrução de identidade diante da multiplicidade dos aspectos sociais e culturais, em que as referências deixam de ser somente das estruturas externas. A complexidade tecnológica crescente tem demandado o desenvolvimento mais amplo e, ao mesmo tempo, profundo, das capacidades humanas no que se refere ao pensar, ao sentir e ao agir sobre o entorno (FARAH et al., 2009; BERBEL, 2011).

Com o avanço tecnológico, faz-se necessário dominar os diversos recursos existentes que atingem todos os âmbitos e permeiam a vida dos indivíduos nas mais variadas faixas etárias. Nessa perspectiva, aqueles que não utilizam ou não têm acesso a essas tecnologias, por vezes, acabam sendo excluídos desses processos de mudanças. Sendo assim, o letramento digital é uma possibilidade que remete ao entendimento sobre a tecnologia, a informática e a comunicação. $\mathrm{O}$ aprendizado tecnológico e a linguagem digital possibilita agir nesses espaços e com outros indivíduos que estão inseridos (FARAH et al., 2009; ZENI et al., 2013; LIMA; ALMEIDA, 2015; PAULOZZO, 2015).

Diante do cotidiano que demanda um contato mais próximo com os diversos aparelhos eletrônicos para desempenhar as atividades rotineiras, os idosos veem a necessidade de se inserirem no universo digital para poderem conviver com a tecnologia e usufruir dos benefícios por ela proporcionados. Contextualizar o público idoso que está inserido neste cenário também se faz importante (BIZELLI et al., 2009; FARAH et al., 2009; MÜLLER, 2012; SILVA, 2014). 
Ao usar a tecnologia, os idosos são ao mesmo tempo atores, produtores e realizadores de suas ações. Os idosos têm a possibilidade de explorar os próprios interesses, de manter a autonomia, de exercer a cidadania, de localizar outros mundos, de cruzar fronteiras geográficas, de estabelecer contatos com outras gerações, bem como com a família e amigos, de estimular a mente, de descobrir e ampliar o conhecimento (FARAH et al., 2009; CHATFIELD, 2012; ZENI et al., 2013).

Por meio da tecnologia computacional, ao aprender, os idosos se atualizam, se informam, se divertem, se interagem, se comunicam, se integram e se inserem socialmente. A tecnologia pode ser capaz de transformar, de certo modo, a realidade dos idosos e de dar novo sentido em suas vidas, na medida, redimensiona seus próprios futuros. De forma participativa, os idosos continuam a opinar e a fazer a história que é construída e registrada constantemente (VALENTE, 2001; FARAH et al., 2009; MÜLLER, 2012).

Contudo, por vezes, os idosos são estereotipados e discriminados por não dominarem o conhecimento digital, na qual se conectam pessoas e sistemas. No início, os idosos sentem-se inseguros por não estarem habituados à tecnologia em questão (FARAH et al., 2009; MÜLLER, 2012; SILVA, 2014).

Ao usarem uma tecnologia de ponta, os idosos quebram paradigmas socioculturais de que velho é passado e não se renova. A idade não é empecilho para a pessoa que quer aprender, é possível aprender durante toda a vida, apenas de formas diferentes. A aprendizagem é construída, o treinamento é mais lento, fragmentado e mediado verbalmente, no início, e aos poucos é substituído pelo desempenho rápido e automatizado. Os idosos são capazes de aprender o novo, de se organizarem para a realização de projetos, de vencer os medos e os desafios. Esses mesmos personagens continuam capazes de se manterem ativos, dadas suas condições físicas e psicológicas (VALENTE, 2001; DOLL; BUAES, 2009; FARAH et al., 2009; MÜLLER, 2012).

Para tanto, é necessário prover oportunidades que tornem o idoso um aprendiz virtual, fornecendo educação, estimulação cognitiva, interação e bem estar. Por meio do letramento digital, o idoso poderá ter mais autonomia e independência na vida cotidiana e uma atuação mais ativa e participativa nas relações sociais (ZENI et al., 2013; LIMA; ALMEIDA, 2015). 
Com isso, o presente estudo teve por objetivos identificar as motivações dos idosos para aprender informática e analisar as estratégias e práticas de uso do computador em sala de aula, a presente pesquisa se justifica pela demanda acadêmica e social sobre o letramento digital de idosos.

\section{Método}

Os participantes da presente pesquisa foram idosos que se matricularam no curso de informática voltado especificamente para esse público, no período de 2013 a 2015. Para a realização foi aplicado um protocolo contendo questões objetivas acerca do contato com o computador, interesses em fazer o curso de informática, principais dificuldades ao aprender informática e acessar a internet, estratégias de estudo e, por fim, avaliação do curso quanto aos aspectos didático-pedagógicos, organização das atividades e infraestrutura e recursos audiovisuais. Diante do tratamento e análise dos dados, utilizou-se o programa IBM SPSS (Statistical Package for the Social Sciences), versão 2.0 .

As questões éticas foram cumpridas de acordo com a Resolução №. 196, que regulamenta as pesquisas envolvendo seres humanos, principalmente no que se refere à explicação da pesquisa, ao esclarecimento de dúvidas preexistentes e à assinatura e o consentimento dos participantes. $O$ estudo foi submetido e aprovado pelo Comitê de Ética e Pesquisa (CAAE: 51343415.8.0000.5409).

\section{RESULTADOS E DISCUSSÃO}

O presente enxerto apresenta dados relativos aos interesses, expectativas e objetivos dos idosos ao participarem de um curso de informática, bem como as principais dificuldades e estratégias de ensino para aprenderem modalidade. Ressalta-se que os idosos participantes do curso de informática assinalaram mais de uma alternativa em cada variável.

Diante dos interesses, expectativas e objetivos dos idosos em relação ao curso de informática, a tabela 1 mostra a frequência de tais dados. 
Tabela 1. Interesses, expectativas e objetivos em relação ao curso de informática, 2013 a 2015.

\begin{tabular}{lr}
\hline \multicolumn{1}{c}{ Variáveis / Categorias } & Frequência \\
\hline Interesses e motivações & 20 \\
Para ter mais conhecimento e se informar & 18 \\
Para utilizar o computador de forma autônoma e independente & 14 \\
Para se socializar com familiares e amigos & 11 \\
Aprimorar o conhecimento sobre informática & 6 \\
Por curiosidade & 5 \\
Para saber utilizar somente a internet & 4 \\
Gosto de tecnologia & 4 \\
Preciso para o meu trabalho & 4 \\
Para fazer novos amigos & \\
Expectativas e objetivos & 17 \\
Ter mais conhecimento e se atualizar & 15 \\
Aprender a usar o computador & 5 \\
Resolver problemas de cunho digital & 4 \\
Interagir socialmente por meio da internet & 1 \\
Exercitar a memória & \\
\hline
\end{tabular}

A tabela 1 apresenta que as motivações mais frequentes dos idosos ao participarem de um curso de informática referem a ter mais conhecimento e se informar, e utilizar o computador de forma autônoma e independente, seguidas das categorias socializar com familiares e amigos e aprimorar o conhecimento sobre informática. A curiosidade e o saber utilizar somente a internet são motivações indicadas pelos idosos. Por fim, o gostar de tecnologia, o precisar para o trabalho e o querer fazer novos amigos também estiveram presentes como motivações para participar de um curso de informática.

Sobre as expectativas e os objetivos em relação ao curso de informática, ter mais conhecimento e se atualizar foi a categoria mais frequente, seguida de aprender a usar o computador. Resolver problemas de cunho digital, interagir socialmente por meio da internet e exercitar a memória também foram assinaladas pelos idosos participantes.

Estudos corroboram aos achados da presente pesquisa sobre as motivações quanto o uso das ferramentas e tecnologias de informação e comunicação, sobretudo para comunicar e interagir, como também para permanecerem autônomos, estarem atualizados e terem uma participação mais ativa (LOLLI; MAIO, 2015; PATRÍCIO; OSÓRIO, 2015; SOARES; ISTOE, 
2015).

A pesquisa de Ordonez e Cachioni (2009) identificou e classificou as motivações dos idosos aos frequentarem a Universidade Aberta à Terceira Idade em aumentar conhecimentos, em primeiro lugar; em segundo, aumentar o contato social; seguido em terceiro por investir no aperfeiçoamento pessoal e, em quarto, ocupar o tempo livre.

Ao participarem de um curso de informática, os idosos desta pesquisa apresentam diversas dificuldades relacionadas ao ensino dessa modalidade, como verificao na tabela 2.

Tabela 2.Principais dificuldades ao aprender informática e internet, 2013 a 2015.

Variáveis / Categorias $\quad$ Frequência

\section{Dificuldades ao aprender informática}

Mexer com fotos e suas propriedades $\quad 15$

Acessar os menus de contexto dos programas 13

Utilizar aplicativos para executar músicas e vídeos 11

Mover arquivos e pastas $\quad 10$

Salvar e mover arquivos $\quad 8$

Imprimir arquivos $\quad 5$

Ativar programas no Menu Iniciar 4

Operações básicas com o equipamento 2

$\begin{array}{ll}\text { Todas as dificuldades apresentadas } & 10\end{array}$

Dificuldades no aprendizado da internet

Navegação 11

Favoritos 10

Fazer pesquisas e downloads 9

E-mails e tarefas específicas 4

Todas as dificuldades apresentada 10

Nenhuma dificuldade apresentada 6

As principais dificuldades ao aprender informática foram mexer com fotos e suas propriedades, seguidas de acessar os menus de contexto dos programas e utilizar aplicativos para executar músicas e vídeos. Propriedades dos arquivos e pastas e salvar e mover arquivos também são dificuldades frequentes, seguidas de imprimir arquivos, ativar programas no Menu Iniciar e fazer operações básicas com o equipamento. Alguns participantes assinalaram ter dificuldades em todas as alternativas apresentadas. 
No que se refere às dificuldades no aprendizado da internet, a navegação em si e as opções dos favoritos foram as mais frequentes, seguidas de fazer pesquisas e downloads. Acessar os e-mails e tarefas específicas também foi assinalado. Alguns idosos apontaram todas as dificuldades apresentadas no que diz respeito ao aprendizado da internet. Porém, outros participantes assinalaram não terem dificuldade sobre essa modalidade.

Desse modo, evidencia-se que as dificuldades dos idosos são distintas. O estudo de Lamarca e colaboradores (2015) revelou que o grupo de idosos teve mais facilidade ao aplicar as ferramentas básicas para o seu manuseio do computador em relação à planilha eletrônica e apresentação de slides. Diferentemente de outras pesquisas que detectaram como principais problemas no uso das tecnologias de informação e comunicação para os mais idosos a digitação, a impressão de documentos, o manuseio do mouse, o uso da internet, o acesso ao e-mail, a comunicação e baixar arquivos; bem como dificuldades quanto à acessibilidade e relacionadas ao foro cognitivo, de falta de concentração, atenção, memória (GOULART; STOBÄUS; MOSQUERA, 2012; LOLLI; MAIO, 2015; PATRÍCIO; OSÓRIO, 2015).

Diante das dificuldades apresentadas, a tabela 3 mostra se os idosos participantes de um curso de informática adotam estratégias de estudos, utilizam métodos e, por vezes, dedicam um tempo diário à informática fora da sala de aula.

Tabela 3. Estratégias, método e tempo de estudo de informática, 2013 a 2015.

\begin{tabular}{lr}
\hline \multicolumn{1}{c}{ Variáveis / Categorias } & Frequência \\
\hline Define estratégias de estudo & \\
Sim & 16 \\
Não & 7 \\
& \\
Método de estudo & 23 \\
Fazendo anotações & 23 \\
Segue as instruções da professora & 16 \\
Pratica em casa & 7 \\
Pratica em outro computador & 5 \\
Não pratica em casa & 0 \\
Apenas escuto as instruções da professora &
\end{tabular}




\section{Tempo diário dedicado ao computador}

Menos de 1 hora

16

Entre 1 e 3 horas

9

Entre 3 e 5 horas

0

Até 10 horas

Dos idosos que responderam, doze assinalaram que definem uma estratégia de estudo e sete não adotam métodos de estudo. Sobre os métodos de estudo, os idosos frequentemente fazem anotações e seguem as instruções da professora. Alguns praticam em casa ou em outro computador. Já outros idosos assinalaram não praticar em casa. Nenhum participante assinalou apenas escuto as instruções da professora.

Boruchovitch (1999) descreve diversas estratégias existentes para o ensino-aprendizagem e destaca que os procedimentos adotados são escolhidos pela pessoa com o intuito de facilitar própria aquisição, armazenamento e utilização da informação e ou tarefa. A aprendizagem do uso das tecnologias também é individualizada, dependendo de cada participante, fazendo com que cada um seja a protagonista de sua própria construção de conhecimento e aprenda em seu ritmo, em uma contínua transformação, tanto de si como da sociedade em que se desenvolve (GOULART; STOBÄUS; MOSQUERA, 2012).

O contato com a informação, o modo como esta será compreendida vai depender diretamente do nível particular de motivação e interesse. Nesse processo, no ambiente digital, o professor se faz importante para apoiar e incentivar a exploração, a experimentação, a interpretação e a seleção sobre o conteúdo. Loreto e Ferreira (2014) acrescentam que é preciso bom relacionamento entre o professor e seus alunos, pois isso, frequentemente, é um fator que motiva a frequência nas aulas e o desempenho no processo de aprendizado.

Quanto ao tempo diário dedicado ao computador, a maior parte dos idosos assinalou ficar menos de uma hora. Outros marcaram despender entre uma e três horas diárias no computador. Segundo a tabela 3 , nenhum idoso fica entre três e cinco horas e apenas um participante fica até dez horas no computador. O estudo realizado por Frias e colaboradores (2011), com um grupo de idosos, identificou que a maioria utiliza o computador diariamente por 
até duas horas; seguido por idosos que utilizam em até quatro horas e a minoria usa por mais de dez horas.

Por fim, a tabela 4 apresenta a avaliação dos idosos ao participarem de um curso de informática.

Tabela 4. Avaliação do curso de informática de acordo com os participantes idosos, 2013 a 2015.

\begin{tabular}{|c|c|c|}
\hline Variáveis/Categoria & & $\mathrm{n}(\%)$ \\
\hline \multicolumn{3}{|l|}{ Aspectos didático-pedagógicos } \\
\hline \multirow[t]{2}{*}{ Empatia e afinidade $(n=17)$} & Ótimo & $15(88)$ \\
\hline & Bom & $2(12)$ \\
\hline \multirow[t]{3}{*}{ Situações problemas $(n=16)$} & Ótimo & $5(31)$ \\
\hline & Bom & $4(25)$ \\
\hline & $\mathrm{NA}^{*}$ & $7(44)$ \\
\hline \multirow[t]{2}{*}{ Material didático-pedagógico $(n=18)$} & Ótimo & $10(56)$ \\
\hline & Bom & $8(44)$ \\
\hline Participação do professor $(n=18)$ & Ótimo & $18(100)$ \\
\hline \multicolumn{3}{|l|}{ Organização das atividades } \\
\hline Pertinência, atualidade e inovação da temática $(n=16)$ & $\begin{array}{l}\text { Ótimo } \\
\text { Bom }\end{array}$ & $\begin{array}{r}10(63) \\
6(37)\end{array}$ \\
\hline \multirow{6}{*}{$\begin{array}{l}\text { Organização e distribuição do conteúdo programático } \\
(\mathrm{n}=15) \\
\text { Adequação dos recursos educacionais às atividades } \\
(\mathrm{n}=16) \\
\text { Horário e pontualidade }(\mathrm{n}=15)\end{array}$} & Ótimo & $8(53)$ \\
\hline & Bom & $7(44)$ \\
\hline & Ótimo & $9(56)$ \\
\hline & Bom & $7(44)$ \\
\hline & Ótimo & $13(87)$ \\
\hline & Bom & 2 (13) \\
\hline \multicolumn{3}{|l|}{ Infraestrutura e recursos audiovisuais } \\
\hline \multirow[t]{3}{*}{ Instalações físicas da sala de aula ( $n=17)$} & Ótimo & $9(56)$ \\
\hline & Bom & $7(38)$ \\
\hline & Regular & $1(6)$ \\
\hline \multirow{2}{*}{ Acessibilidade e funcionalidade $(n=17)$} & Otimo & $8(44,4)$ \\
\hline & Bom & $9(50)$ \\
\hline \multirow[t]{3}{*}{ Recursos audiovisuais $(n=16)$} & Ótimo & $9(56)$ \\
\hline & Bom & $5(31)$ \\
\hline & Regular & $2(13)$ \\
\hline \multirow[t]{3}{*}{ Comunicação, informações e atendimento $(n=17)$} & Otimo & $11(65)$ \\
\hline & Bom & $5(29)$ \\
\hline & Regular & $1(6)$ \\
\hline
\end{tabular}

${ }^{*} \mathrm{NA}=\mathrm{Não}$ se aplica

No que se refere aos aspectos didático pedagógico, as categorias empatia e afinidade e material didático foram avaliadas como ótimas e boas. Em situações problemas a maioria assinalou não se aplica, outros avaliaram como ótimo e bom. Sobre a participação do professor houve unanimidade ao avaliarem como ótima. 
Quanto à variável organização das atividades, a maioria dos participantes avaliaram as categorias pertinência, atualidade e inovação da temática, organização e distribuição do conteúdo programático, adequação dos recursos educacionais às atividades e horário e pontualidade como sendo ótimas e boas.

Por fim, sobre a infraestrutura e recursos audiovisuais, os idosos avaliaram a acessibilidade e funcionalidade em ótimo e bom. As categorias instalações físicas da sala de aula, recursos audiovisuais e comunicação, informação e atendimento foram avaliados como sendo ótimos, bons e regulares. Vale destacar que essa variável se refere ao local onde o curso é desenvolvido.

De modo geral, o curso de informática segundo os participantes idosos é avaliado como ótimo e bom, expressando total satisfação. Na pesquisa de Ordonez e Cachioni (2009) realizada com idosos matriculados na Universidade Aberta à Terceira Idade, grande parte dos entrevistados declarou "satisfeita" e "muito satisfeita" com seu envolvimento no programa, sugerindo que os objetivos foram atingidos de modo eficaz às necessidades suscitadas.

\section{CONSIDERAÇÕES FINAIS}

Em sociedades cada vez mais digitalizadas, ao qual o avanço é iminente e a tecnologia se faz presente em quase todos os contextos, para continuarem autônomas e participativas, as pessoas precisam se manter atualizadas constantemente. A busca por conhecimento, aperfeiçoamento e treinamento duradouro, para manusear essas ferramentas, se faz necessária, especialmente para aqueles, como os idosos, que nasceram dessa ampliação tecnológica.

Diante dos dados apresentados, o curso de informática segundo os participantes é avaliado como ótimo e bom, expressando total satisfação. As motivações, as expectativas e os objetivos dos idosos ao participarem de uma oficina de informática exclusiva para esse público estão voltados justamente para a atualização tecnológica e aquisição de conhecimento nesta área. As dificuldades assinaladas ainda são pontuais e correlacionadas ao manuseio do computador, ao entendimento e segurança sobre a tarefa e à necessidade de repetição e prática. Evidenciou-se que poucos se dedicam diariamente à 
atividade de informática fora da sala de aula, ora por não terem o equipamento ou mesmo não terem tempo, ora por não estarem totalmente seguros executarem as tarefas.

Uma vez que o modo de ensino-aprendizagem de informática para idosos é heterogêneo, observado na facilidade de alguns e na dificuldade de outros, recomenda-se mais investigações sobre o assunto, principalmente no que se refere ao processo de aprendizado e às estratégias que os idosos adotam nesta modalidade. Ressalta-se, por fim, que o registro na literatura sobre a temática ainda é escasso mesmo sendo notória a interferência da tecnologia no âmbito social dos idosos.

\section{REFERÊNCIAS}

BERBEL, Neusi Aparecida Navas. As metodologias ativas e a promoção da autonomia de estudantes. Semina: Ciências Sociais e Humanas, Londrina, vol. 32, n. 1, p. 25-40, 2011.

BORUCHOVITCH, Evely. Estratégias de aprendizagem e desempenho escolar: considerações para a prática educacional. Psicol. Reflex. Crit., vol.12 n.2, Porto Alegre. 1999.

CHATFIELD, Tom (Tradução: FIUZA, Bruno). Como viver na era digital. Editora: Objetiva, 2012, p.176.

DOLL, Johannes; BUAES, Caroline Stumpf. Aprendizagem em cursos de inclusão digital para pessoas adultas e idosas. RBCEH, Passo Fundo, vol. 6, n. 3, p. 320-331. 2009.

FARAH, R. M. et al.. Novas Tecnologias no Envelhecimento. Revista Kairós Gerontologia, Caderno Temático 5, vol. 12, p.128-172. 2009.

FRIAS, Marcos Antonio da Eira et al. Utilização de ferramentas computacionais por idosos de um centro de referência e cidadania do idoso. Rev. Esc. Enferm. USP, vol.45, n.spe, pp.1606-1612. 2011.

GOULART, Denise; STABÄUS, Claus Dieter; MOSQUERA, Juan José Mouriño. Inclusão digital na adultez tardia e o reencantamento da aprendizagem. In: FERREIRA, Anderson Jackle et. Al. (org.). Educação \& envelhecimento - Dados eletrônicos. Porto Alegre: EdiPUCRS, p.79-94. 2012. 
LAMARCA, Daniel Sá Freire et al. TIC presente nas universidades: uma análise no projeto de extensão Universidade Aberta à Terceira Idade (UNATI). 8을 Congresso de Extensão Universitária da UNESP, 2015.

LIMA, Samuel de Carvalho; ALMEIDA, Lúrya Valéria de Oliveira Sousa. Letramento Digital de Idoso no contexto da EJA em Mossoró-RN. Tear: Revista de Educação Ciência e Tecnologia, Canoas, vol.4, n.1, 2015. LOLLI, Maria Carolina Gobbi dos Santos; MAIO, Eliane Rose. Uso da tecnologia para idosos: perfil, motivações, interesses e dificuldades. Revista Educação, Cultura e Sociedade. Sinop/MT/Brasil, vol. 5, n. 2, p. 211-223, jul./dez. 2015.

LORETO, Elisa Sergi Gordilho; FERREIRA, Giselle Martins dos Santos. Desafios e possibilidades para a Inclusão Digital da Terceira Idade. Revista Eletrônica de Educação, vol. 8, n. 2, p. 120-137, 2014.

MÜLLER, Daniele. O Envelhecimento e a Inclusão Digital de Idosos. Universidade Regional do Noroeste do Estado do Rio Grande Do Sul. 2012. ORDONEZ, Tiago Nascimento; CACHIONI Meire. Universidade aberta à terceira idade: a experiência da Escola de Artes, Ciências e Humanidades. RBCEH, Passo Fundo, vol. 6, n. 1, p. 74-86, jan./abr. 2009.

PATRÍCIO, Maria Raquel; OSÓRIO, Antonio. Inclusão Digital com Aprendizagem Intergeracional. Challenges 2015: Meio Século de TIC na Educação. Biblioteca Digital Instituto Politécnico de Bragança, p.795-809. 2015.

PAULOZZO, Marília Degasperi. Experiência com Informática para pessoas da Terceira Idade. Universidade Estadual Paulista "Júlio de Mesquita Filho". Instituto de Biociência. Rio Claro. 2015.

SILVA, T. R. et al. Inclusão Digital da Terceira Idade. Anais Congresso Sul Brasileiro de Computação, vol. 7. 2014.

SOARES, Márcia Regina Pacheco; ISTOE, Rosalee Santos Crespo.

Alfabetização e Inclusão de Pessoas Idosas: uma proposta interdisciplinar mediada pelas tecnologias da informação e da comunicação. Revista

Científica Interdisciplinar. № 3, vol. 2, artigo № 17, julho/setembro 2015. 
VALENTE, José Armando. Aprendizagem Continuada ao longo da Vida: o exemplo da terceira idade. KACHAR, Vitória (Org.) Longevidade: um novo desafio para a educação. São Paulo: Cortez Editora, 2001, p.27-44.

ZENI, J. et al. Inclusão Digital - Informática Terceira Idade. Seminário de Extensão Universitária da Região Sul. 2013. 


\section{TRALLO, Roberta dos Santos Tarallo}

Gerontóloga e Mestre em Gerontologia pela FCM/UNICAMP. Doutoranda em Educação na FE/UNICAMP. Professora terceirizada do Curso do Programa de Formação e Educação Continuada do Idoso - PROFECI da Faculdade de Jaguariúna.

Link do Currículo Lattes: http://lattes.cnpq.br/7626320912350132

\section{SÉ, Elisandra Villela Gasparetto}

Fonoaudióloga formada pela Faculdade Integradas Teresa D’Ávila de Lorena FATEA (1995). Mestre em Gerontologia - Faculdade de Educação FE/UNICAMP (2003). Doutora em Linguística - Instituto de Estudos da Linguagem - Área de Neurolinguística - IEL/UNICAMP (2011). Especialista em Educação em Saúde para Preceptores do SUS pelo Instituto Sírio Libanês de Ensino e Pesquisa - IEP/HSL (2014). Exerceu a função de pesquisadora colaboradora no Ambulatório de Psiquiatria Geriátrica do Hospital de Clínicas FCM/UNICAMP (2000-2012), e no Ambulatório de Geriatria e Gerontologia HC/FCM/UNICAMP (2007-2010). Foi pesquisadora colaboradora no Centro de Convivência de Afásicos - IEL/UNICAMP (2006-2011). Foi Presidente da Associação Brasileira de Alzheimer - Regional São Paulo, gestão 2012-2014. Coordenadora da Associação Brasileira de Alzheimer - ABRAz Sub-regional Campinas/SP e da Sub-regional de Jaguariúna/SP. Foi pesquisadora visitante na Associação Alzheimer Portugal - Lisboa (2013). Foi membro conselheiro do Conselho Municipal de Saúde de São Paulo (2012 - 2014), e do Conselho Municipal do Idoso de Jaguariúna (2012 - 2013). Atualmente é membro do Conselho Municipal do Idoso de Jaguariúna. Diretora do Centro Educacional Perspectiva, Cursos de Pós-graduação e extensão com parceria Grupo Polis Educacional (Faculdade de Jaguariúna e Faculdade Max Planck de Indaiatuba). Idealizadora e Coordenadora do Programa de Formação e Educação Continuada do Idoso da Faculdade Jaguariúna e da Faculdade Max Planck, Indaiatuba - SP. Coordenadora do Comitê de Ética em Pesquisa de Seres Humanos da Faculdade de Jaguariúna e da Faculdade Max Planck. Membro do Comitê de Ética em Pesquisa do Uso de Animais da FAJ e da Faculdade Max Planck. Conselheira do Conselho Municipal do Idoso de Jaguariúna. Membro Conselheira e Pesquisadora do Núcleo de Estudo e Pesquisas Interdisciplinares da Faculdade de Jaguariúna e da Faculdade Max Planck. Membro parecerista da Revista Intellectus. Professora Titular da Universidade Paulista - UNIP É colunista semanal do site Vya Estelar da UOL. Coluna Mente na Terceira Idade. Link do Currículo Lattes: http://lattes.cnpq.br/1267773065197502 